\title{
Increased expression of $\alpha$-actinin- 4 is associated with unfavorable pathological features and invasiveness of bladder cancer
}

\author{
HIDEHIKO YOSHII, KEIICHI ITO, TAKAKO ASANO, AKIO HORIGUCHI, \\ MASAMICHI HAYAKAWA and TOMOHIKO ASANO
}

Department of Urology, National Defense Medical College, Tokorozawa, Saitama 359-8513, Japan

Received April 19, 2013; Accepted June 7, 2013

DOI: 10.3892/or.2013.2577

\begin{abstract}
In the present study, the association between clinicopathological parameters and $\alpha$-actinin-4 (ACTN4) expression in bladder cancer specimens was evaluated, and the functional role of ACTN4 in bladder cancer cells was investigated. Immunohistochemistry using anti-ACTN4 antibody was performed in bladder cancer specimens (53 superficial and 42 muscle-invasive cases) from 95 patients who underwent radical cystectomy $(n=46)$ or transurethral resection (TUR) only ( $\mathrm{n}=49$ ). We divided the levels of ACTN4 expression into 2 groups (low or high) by comparing the staining intensity in each specimen with that of the vascular endothelial cells in the same specimen, and we evaluated the correlations between these levels and pathological parameters, recurrence and prognosis. We also investigated the effects of ACTN4 suppression by siRNA on the invasive ability and proliferation of T24 and KU19-19 cells. High ACTN4 expression was significantly associated with higher tumor grade and higher pT stage. In patients with superficial bladder cancer treated only by TUR, the rate of intravesical recurrence did not differ significantly between patients with high ACTN4 expression and patients with low ACTN4 expression. In patients who had muscle-invasive tumors and underwent radical cystectomy, high ACTN4 expression was associated with neither recurrence nor poor prognosis. Nonetheless, high ACTN4 expression was shown by a large percentage $(81 \%)$ of patients with muscle-invasive bladder cancer and by a small percentage (17\%) of patients with superficial bladder cancer. Furthermore, the leading edges of the invasive bladder cancer showed increased ACTN4 expression. ACTN4 suppression significantly reduced the number of invading bladder cancer cells but unexpectedly increased the proliferation of bladder cancer cells. ACTN4 suppression increased the phosphorylation of ERKs but not AKT or STAT3, suggesting that the increased proliferation
\end{abstract}

Correspondence to: Dr Keiichi Ito, Department of Urology, National Defense Medical College, 3-2 Namiki, Tokorozawa, Saitama 359-8513, Japan

E-mail: itok@ndmc.ac.jp

Key words: bladder cancer, $\alpha$-actinin-4, urothelial carcinoma, invasion, proliferation, ERKs due to ACTN4 suppression was mediated in part by the ERK pathway. ACTN4 expression may suppress the proliferation of bladder cancer cells and may produce conditions which facilitate cancer cell invasion.

\section{Introduction}

Bladder cancer is the most frequently occurring urological malignancy. The prognosis for patients with non-invasive bladder cancers is generally good, and most non-muscle invasive bladder cancer can be controlled by transurethral resection (TUR) of the tumor combined with intravesical instillation of cytotoxic agents or Bacillus Calmette-Guerin (BCG). Patients with invasive bladder cancer, in contrast, particularly those with muscle-invasive disease, face the possibility of postoperative distant metastasis or local recurrence even after radical cystectomy, and for those patients the recurrence rate is positively correlated with increased tumor stage ( $T$ stage) and histological grade $(1,2)$. The prognosis is particularly poor for patients with lymph node metastasis that is histologically confirmed in radical cystectomy specimens; the 5-year overall survival rate is only $23-35 \%$ (1-3). Furthermore, patients with distant metastasis have a reduced survival rate (4).

Although cisplatin-based chemotherapy is effective for patients with metastatic bladder cancer, patients are rarely cured by chemotherapy and long-term survival is rare. The 5 -year overall survival of chemotherapy-treated patients with metastatic disease is reportedly less than $20 \%(5,6)$. The most effective way to improve the prognosis of patients with bladder cancer is to diagnose the disease early and to operate immediately. If recurrence after cystectomy could be predicted, adjuvant chemotherapy could be administered when the lesions are small. Thus, biomarkers that predict the recurrence of bladder cancer need to be found.

We previously identified bladder cancer antigens recognized by the $\operatorname{IgG}$ antibody in patients with advanced bladder cancer using the serological identification of tumor antigens by cDNA expression cloning (SEREX) method (7). This method has identified tumor antigens that are recognized by $\mathrm{CD}^{+}$ T cells and are possible immunotherapy targets, but it could also be used to identify antigens that are overexpressed in cancer cells and can be used as diagnostic or prognostic biomarkers. The SEREX method has previously identified prognostic biomarkers such as p53, galectin-3 and NY-ESO-1 $(8,9)$. In a 
previous study (7) we found that the serum of a patient with metastatic bladder cancer contained IgG antibodies that recognized $\alpha$-actinin-4 (ACTN4). We therefore speculated that IgG antibodies to ACTN4 may be produced in response to the ACTN4 overexpressed in bladder cancer cells.

The $\alpha$-actinins (ACTNs) are a family of actin-binding proteins and are involved in cytoskeletal reorganization. Four isoforms of human ACTN have been identified. ACTN1 and ACTN4 are nonmuscle proteins thought to cross-link and connect the actin filaments and connect the actin cytoskeleton to the cell membrane (10). The two non-muscle ACTNs may bind different targets. Immunohistochemical analysis found ACTN1 to be localized at the interface between actin stress fibers and plasma membrane adherens junctions, whereas ACTN4 appears to cross-link actin stress fibers (10). ACTN4 is highly concentrated at the leading edges of motile cells and in cytoplasmic regions with sharp cell extensions (10). ACTN4 was found to be preferentially localized in moving structures, such as the dorsal ruffles of macrophages (11). ACTN4 is reportedly associated with invasion and metastasis of cancer cells in several malignant tumors. Its increased expression in the cytoplasm and/or cell membrane is related to the prognosis of patients with breast cancer $(10,12)$ and non-small cell lung cancer (13), and overexpression of ACTN4 in colon cancer cells was found to lead to increased lymph node metastasis in an animal model (14). ACTN4 has recently been reported to be involved in the invasive activity of bladder cancer cells (15). Yet, neither the association of tissue ACTN4 expression to clinicopathological factors, nor the impact of ACTN4 expression on recurrence or prognosis has been fully evaluated in human bladder cancer specimens.

In the present study, we evaluated the expression of ACTN4 in bladder cancer specimens and evaluated the correlation between ACTN4 expression level and recurrence and prognosis. We also examined the effects of ACTN4 knockdown on the invasive ability and proliferation of cultured bladder cancer cells.

\section{Materials and methods}

Cell culture and reagents. The human bladder cancer cell lines used in this study were T24 (16) and KU19-19 (17). T24 cells were purchased from ATCC (American Type Culture Collection, Manassas, VA, USA), and KU19-19 cells were kindly provided by the Urology Department of Keio University (Shinjuku, Tokyo, Japan). These cell lines were maintained in RPMI-1640 (Invitrogen Life Technologies, Carlsbad, CA, USA) supplemented with $10 \%$ fetal bovine serum (FBS), 100 IU/ $\mathrm{ml}$ penicillin and $100 \mu \mathrm{g} / \mathrm{ml}$ streptomycin (Invitrogen Life Technologies). Antibodies against ACTN4 (polyclonal; Alexis Biochemicals, San Diego, CA, USA), phospho-Akt (Ser473), Akt, phospho-STAT3, STAT3, phospho-ERKs, ERKs (Cell Signaling Technology, Inc., Boston, MA, USA) and $\beta$-actin (Millipore, Billerica, MA, USA) were used. Horseradish peroxidase-conjugated secondary antibodies and an enhanced chemiluminescence system (Amersham Pharmacia Biotech, Piscataway, NJ, USA) were also used.

Patients. The clinicopathological features of the patients who underwent surgical intervention (TUR and/or radical cystectomy) for urothelial carcinoma of the bladder at our institution between 1994 and 2007 were carefully reviewed according to clinical records and pathological reports. Patients with pure bladder squamous cell carcinoma and patients with pure adenocarcinoma were excluded from this study. Patients without sufficient follow-up data and/or without urothelial carcinoma histology were also excluded. We evaluated paraffin-embedded sections of surgical specimens obtained from 95 patients. Among the 95 patients, 49 patients with superficial bladder cancer who underwent TUR between 1994 and 2000 were randomly selected. In addition, 46 patients who underwent radical cystectomy at our institute between 1997 and 2007 were reviewed and enrolled as participants. The 95 participating patients included 78 men and 17 women whose ages ranged from 29 to 87 years (median age, 68); 42 had invasive bladder cancer and 53 had superficial disease. Forty-six of these 95 patients underwent radical cystectomy after TUR.

The 49 patients who underwent TUR had only urothelial carcinoma and one of these patients had some component of adenocarcinoma in addition to urothelial carcinoma. The histological diagnoses of all 46 patients who underwent radical cystectomy were urothelial carcinoma. Five of these patients had squamous cell carcinoma components and one had an adenocarcinoma component. The pathological tumor stage and histological grade were determined according to the 2009 TNM classification system (7th edition). The predominant histological grade of 21 tumors was grade 1 , that of 40 tumors was grade 2, and that of 34 tumors were grade 3 . Fifty-two $(54.7 \%)$ of the tumors had some degree of grade 3 components. Thirty-four of the tumors were pTa, 19 were pT1, 18 were pT2, 20 were pT3, and 4 were pT4. Five of the 46 patients $(10.9 \%)$ who underwent radical cystectomy had metastases in surgically resected lymph nodes, and 35 of the 46 patients $(76.1 \%)$ had lymphovascular invasion (LVI).

To monitor intravesical recurrence in the patients with superficial bladder cancer, the patients were evaluated postoperatively by cystoscopy every 3 months for the first 2 years and every 6-12 months thereafter. To monitor the occurrence of local and/or distant metastases in the patients who underwent radical cystectomy, they were evaluated postoperatively every 3-6 months for the first 5 years and every 6-12 months thereafter. Follow-up examinations for the patients after radical cystectomy consisted of physical examination, chest radiography, abdominal and chest $\mathrm{CT}$, blood tests, and if indicated, radionuclide bone scanning. Follow-up intervals were calculated from the date of surgical intervention to the last recorded follow-up. The median follow-up interval was 38.4 months (range 1-153 month) for patients who had superficial bladder cancer and were treated only by TUR, and the median followup interval was 47.7 months (range 3-135 months) for patients who underwent radical cystectomy. Intravesical recurrencefree survival was evaluated using the date at which intravesical recurrence was identified, and extravesical recurrence-free survival was evaluated using the date at which local recurrence or metastatic disease was identified. Cause-specific survival was evaluated using the date of death due to disease progression or the last follow-up date. Disease progression was defined as evidence of recurrence or metastasis on radiological examination or physical examinations. This study was approved by the institutional review board. 
Immunohistochemical analysis and tissue evaluation. Paraffin-embedded sections (5 $\mu \mathrm{m})$ were mounted on slides, deparaffinized in xylene, and rehydrated through graded ethanols. For antigen retrieval, the sections were placed in Dako Target Retrieval Solution High pH (Dako Corp., Carpinteria, $\mathrm{CA}, \mathrm{USA}$ ) and heated at $95^{\circ} \mathrm{C}$ for $50 \mathrm{~min}$. Endogenous peroxidase activity was quenched with Dako peroxidase blocking reagent (Dako Corp.) for $10 \mathrm{~min}$. Sections were incubated in $10 \%$ normal goat serum in phosphate-buffered saline (PBS) for $60 \mathrm{~min}$ at room temperature and then incubated overnight at $4^{\circ} \mathrm{C}$ with primary antibody for ACTN4 (rabbit polyclonal; Alexis Biochemicals) at appropriate dilutions in PBS. They were then stained using the Simple Stain Max PO kit (Nichirei Corp., Tokyo, Japan) according to the manufacturer's instructions. Reaction products were visualized by immersing the slides in diaminobenzidine for $2 \mathrm{~min}$. After the sections were counterstained with hematoxylin, they were covered with glass coverslips. Vascular epithelial cells, which are known to be abundant in ACTN4 (18), served as positive internal controls. Immunostaining results for all tumor sections were evaluated by 2 individuals (H.Y. and K.I.) blinded to all clinical data. Since the anti-ACTN4 antibody clearly stained the vascular endothelial cells, the staining intensity of each tumor section was compared with that of the vascular endothelial cells in the section. Tumors with a staining intensity equal to (level 3) or greater than (level 4) that of the vascular endothelial cells were defined as tumors with high ACTN4 expression, while those with staining intensity less than that of the vascular endothelial cells were defined as tumors with low ACTN4 expression (level 1 or 2). Negative ACTN4 staining was defined as level 1 staining. The percentage of each ACTN4 staining level in each specimen was determined by microscopically reviewing the entire slide at x200 magnification. The predominant level of ACTN4 expression was determined for each patient and defined as the ACTN4 expression level for that patient.

Immunocytochemistry for the leading edges of cultured bladder cancer cells. Cells (T24 and KU19-19) were grown to confluence on 6-well tissue culture plates and a wound was made by scraping in the middle of the cell monolayer with a cell scraper $1.1 \mathrm{~cm}$ wide (Sumitomo Bakelite Co., Ltd., Tokyo, Japan). After floating cells were removed by extensive washing with PBS, fresh complete medium was added. Then cells were incubated for $24 \mathrm{~h}$. Immunocytochemistry was performed to confirm protein expression of ACTN4 in the bladder cancer cells. Twenty-four hours after incubation, cells in 6-well plates were fixed for $5 \mathrm{~min}$ in $4 \%$ paraformaldehyde in PBS. After fixation, cells were washed 3 times ( $5 \mathrm{~min} / \mathrm{wash}$ ) with $0.3 \mathrm{M}$ glycine and then permeabilized in $0.1 \%$ Triton-X in PBS for $7 \mathrm{~min}$. After 3 washes in PBS (5 min/wash), non-specific binding sites were blocked with $10 \%$ normal goat serum (NGS) in PBS for $1 \mathrm{~h}$. Cells were then incubated with $1 \mu \mathrm{g} / \mathrm{ml}$ anti-ACTN4 antibody and 1\% NGS in PBS for $30 \mathrm{~min}$. After 3 washes in PBS (5 min/wash), cells were incubated in a 1:500 dilution of Alexa-Fluor ${ }^{\circledR}$-594-conjugated goat anti-rabbit IgG (Invitrogen Life Technologies) for $30 \mathrm{~min}$ and then washed 3 times in PBS ( $5 \mathrm{~min} /$ wash). The leading edge and other regions of the cultured cells were observed using fluorescence microscopy (x400).

To compare the protein expression of ACTN4 in the leading edge of the cultured bladder cancer cells, western blot analysis was performed. Bladder cancer cells (T24 and KU19-19) were grown to confluence in $10-\mathrm{cm}$ cell culture plates and were scratched by a cell scraper (width, $1.1 \mathrm{~cm}$ ) to create leading edges in the cell colonies. After a 24-h incubation, cells were obtained by scraping them from the leading edge and non-leading edge areas of the colonies on the different plates. These cells were subjected to western blot analysis.

Western blot analysis. Bladder cancer cells were lysed in RIPA buffer containing $10 \mathrm{mM}$ Tris- $\mathrm{HCl}, 150 \mathrm{mM} \mathrm{NaCl}$, $1 \%$ Triton $\mathrm{X}-100,5 \mathrm{mM}$ ethylenediaminetetraacetic acid, $1 \%$ sodium deoxycholate, $0.1 \%$ sodium dodecyl sulfate, $1.2 \%$ aprotinin, $5 \mu \mathrm{M}$ leupeptin, $4 \mu \mathrm{M}$ antipain, $1 \mathrm{mM}$ phenylmethylsulfonyl fluoride and $0.1 \mathrm{mM} \mathrm{Na} \mathrm{VO}_{4}$. Equal amounts of the resulting lysates were separated by using $10 \%$ SDS-PAGE and were then transferred to nitrocellulose membranes. The membranes were blocked with a solution containing 5\% skim milk, and incubated overnight with primary antibodies at $4^{\circ} \mathrm{C}$. They were then incubated with secondary antibodies coupled to horseradish peroxidase (GE Healthcare, Buckinghamshire, UK). The reactive proteins were visualized by enhanced chemiluminescence (Amersham ECL Western blotting detection reagents and analysis system; GE Healthcare) according to the manufacturer's recommendations.

Silencing of ACTN4 by siRNA transfection. T24 and KU19-19 cells were plated in 24-well culture plates 1 day before being transfected with $40 \mathrm{nM}$ ACTN4-specific siRNA (sc-43101) or non-sense siRNA (sc-37007; both from Santa Cruz Biotechnology Inc., Santa Cruz, CA, USA) that had been mixed with Lipofectamine 2000 (Invitrogen Life Technologies) according to the manufacturer's protocol. The cells were then cultured for $48 \mathrm{~h}$ and assayed for ACTN4 expression by western blot analysis, invasion by Matrigel invasion assay, cell viability by MTS assay, and cell growth by cell count.

Matrigel invasion assay. The Matrigel invasion assay was performed using Matrigel-coated invasion chambers (Becton-Dickinson, Franklin Lakes, NJ, USA) as described previously (19). Briefly, a suspension of $5 \times 10^{4}$ cells in $500 \mu \mathrm{l}$ serum-free medium was added to the insert, and $750 \mu \mathrm{l}$ RPMI with $10 \%$ FBS was added to the bottom of the well. After the plates were incubated for $22 \mathrm{~h}$ at $37^{\circ} \mathrm{C}$, the inserts were fixed in methanol, the filters were stained with $1 \%$ toluidine blue in $1 \%$ borax, and the number of cells that invaded through the Matrigel-coated Transwell inserts was counted at x40 magnification. The number of cells was counted in independent triplicate experiments for at least 5 fields/well.

Cell viability assays and cell counts. Cell viability was assessed by MTS assay (Promega Corporation, Madison, WI, USA) according to manufacturer's instructions. Briefly, cells treated with ACTN4 siRNA or non-sense siRNA were incubated for $48 \mathrm{~h}$ in 96 -well plates $\left(5 \times 10^{3}\right.$ cells/well). Two hours after adding MTS, the plates were read at a wavelength of $490 \mathrm{~nm}$ in a microplate autoreader. Results are expressed as the mean optical density of the 6-well set/group, repeated twice with similar results. For cell counts, cells treated with ACTN4 siRNA or non-sense siRNA were incubated for $48 \mathrm{~h}$ in 24 -well plates $\left(1.5 \times 10^{4}\right.$ cells/well in triplicate). The total 
cell number in 3 independent wells/group was counted at the indicated time using a hemocytometer, and the mean value of 4 fields was recorded.

Statistical analyses. All statistical analyses were performed using the StatView 5.0 software package for Windows (SAS Institute Inc., Cary, NC, USA). Results are presented as the means \pm standard deviation. Variables of different groups were compared using the Mann-Whitney U test. The independence of fit of categorical data was analyzed using the Chi-square test. Survival curves were constructed by the Kaplan-Meier method, and differences between them were assessed using the log-rank test. In all tests a p-value $\leq 0.05$ was considered to indicate statistical significance.

\section{Results}

Expression of ACTN4 in bladder cancer specimens. As in a previous study (18), ACTN4 was clearly expressed in vascular endothelial cells (Fig. 1A; level 3 expression). The expression intensity was level 1 in 9 tumors (Fig. 1B), level 2 in 43 tumors (Fig. 1C), level 3 in 34 tumors (Fig. 1D) and level 4 in 9 tumors. High ACTN4 expression (level 3 or 4) was found in 34 of the $42(81 \%)$ specimens from patients with muscle-invasive bladder cancer but only in 9 of the $53(17 \%)$ specimens from patients with superficial bladder cancer. In specimens of patients with muscle-invasive bladder cancer, cells at the leading edges of the invasive tumors (Fig. 2A) and scattered invading cells frequently showed high ACTN4 expression (Fig. 2B-D). Cells aligned at the leading edges of invading bladder cancer sometimes showed higher ACTN4 expression in the cytoplasm than in cells in the non-leading edge areas (Fig. 2A).

Association of ACTN4 expression level with clinicopathological factors. Patients with high ACTN4 expression had significantly higher pathological $\mathrm{T}$ stage $(\mathrm{p}<0.0001)$ and higher histological grade $(\mathrm{p}<0.0001)$ than those with low ACTN4 expression (Table I). A significantly higher percentage of patients with muscle-invasive disease ( $\geq$ pT2) had high ACTN4 expression when compared with patients with a pTa or pT1 tumor (81 vs. $17 \%, \mathrm{p}<0.001$ ). The number of concomitant CIS lesions did not significantly differ between patients with high ACTN4 expression and those with low expression. In patients with superficial bladder cancer who underwent TUR only $(n=49)$ there was no significant difference between the intravesical recurrence-free survival of patients with high ACTN4 expression and that of patients with low expression $(\mathrm{p}=0.2598)$. In patients who underwent radical cystectomy $(n=46)$ neither extravesical recurrence-free survival nor cancer-specific survival differed significantly between patients with high ACTN4 expression and patients with low ACTN4 expression $(\mathrm{p}=0.8804$ for extravesical recurrence-free survival and $p=0.7529$ for cancer-specific survival) (data not shown).

ACTN4 expression in the leading edge of cultured bladder cancer cells. Fig. 3A shows western blot results for ACTN4 in 5 human bladder cancer cell lines. All the cell lines clearly expressed ACTN4, and we used T24 and KU19-19 cells for the following experiments. Immunocytochemistry indicated that
Table I. Characteristics of the 95 patients with bladder cancer.

\begin{tabular}{|c|c|c|c|}
\hline & \multicolumn{2}{|c|}{ ACTN4 } & \multirow[b]{2}{*}{$\mathrm{P}$-value ${ }^{\mathrm{a}}$} \\
\hline & Low $(n=52)$ & High $(n=43)$ & \\
\hline Gender, $\mathrm{n}$ & & & 0.7087 \\
\hline Male & 42 & 36 & \\
\hline Female & 10 & 7 & \\
\hline Age (years) & & & 0.341 \\
\hline$<70$ & 34 & 24 & \\
\hline$\geq 70$ & 18 & 19 & \\
\hline T stage, $\mathrm{n}$ & & & $<0.0001$ \\
\hline $\mathrm{pTa}$ & 32 & 2 & \\
\hline pT1 & 12 & 7 & \\
\hline pT2 & 3 & 15 & \\
\hline pT3 & 2 & 18 & \\
\hline pT4 & 3 & 1 & \\
\hline Muscle invasion $(\geq p T 2), n$ & & & $<0.0001$ \\
\hline Yes & 8 & 34 & \\
\hline No & 44 & 9 & \\
\hline Tumor grade (dominant), $\mathrm{n}$ & & & $<0.0001$ \\
\hline G1 & 18 & 3 & \\
\hline $\mathrm{G} 2$ & 26 & 14 & \\
\hline G3 & 8 & 26 & \\
\hline Presence of CIS, $\mathrm{n}$ & & & 0.3223 \\
\hline Yes & 4 & 6 & \\
\hline No & 48 & 37 & \\
\hline
\end{tabular}

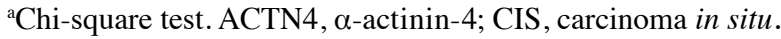

in cultured cells there was more ACTN4 in the leading edge of cells than that in the non-leading edge of cells (Fig. 3C), and western blot analysis showed that ACTN4 expression was stronger in the leading edge of cells (Fig. 3B).

Effect of ACTN4 knockdown on invasive ability. siRNA interference suppressed ACTN4 expression in T24 and KU19-19 cells (Fig. 4A), and we assessed the effect of this suppression on the invasiveness of bladder cancer cells by using Matrigel invasion assays. Matrigel invasion assay demonstrated that the number of T24 and KU19-19 cells invading through the chamber was significantly decreased by ACTN4 suppression (Fig. 4B and C), indicating that ACTN4 may contribute to the invasiveness of bladder cancer.

Effect of ACTN4 knockdown on bladder cancer cell viability and proliferation. We found that ACTN4 suppression significantly increased the viability of the 2 bladder cancer cell lines we investigated (Fig. 5A). We also found that 2 days after T24 and KU19-19 cells were treated with control siRNA or ACTN4 siRNA, the total number of ACTN4-siRNA-treated cells was significantly higher than the number of control-siRNA-treated cells (Fig. 5B and C) $(\mathrm{p}<0.05)$.

Effects of ACTN4 on intracellular signaling pathways of bladder cancer cells. The results of the invasion assay, MTS 

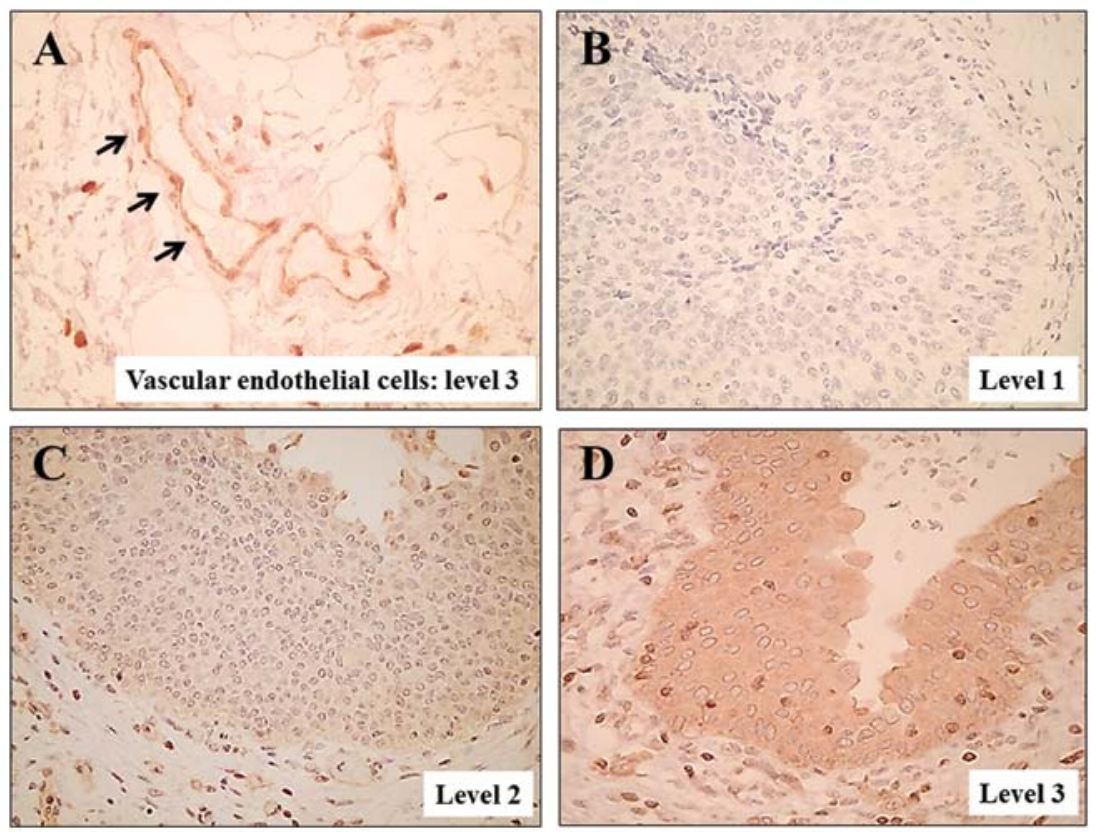

Figure 1. ACTN4 expression in bladder cancer specimens. (A) ACTN4 expression in vascular endothelial cells (black arrows) (level 3 expression). (B) Level 1 expression in bladder cancer (negative or almost no expression). (C) Level 2 expression in bladder cancer (lower expression than in vascular endothelial cells). (D) Level 3 expression in bladder cancer (expression similar to that in vascular endothelial cells).
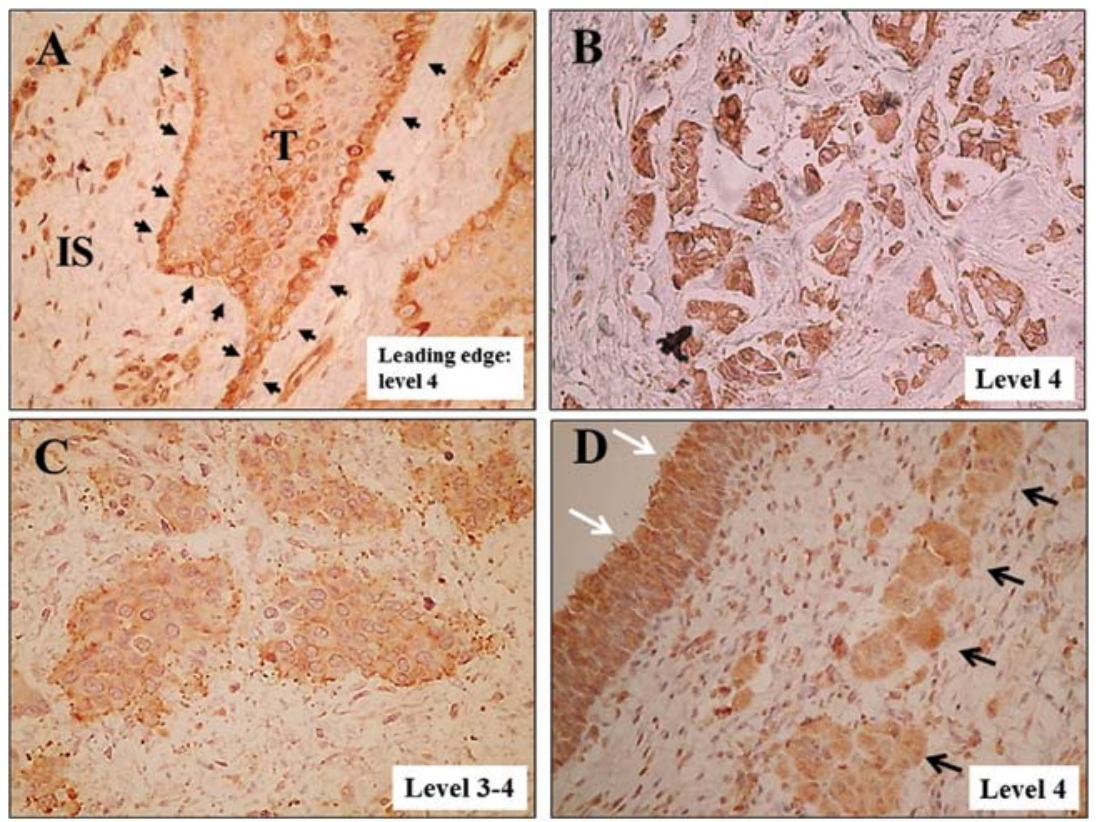

Figure 2. ACTN4 expression in bladder cancer specimens. (A) ACTN4 expression in the leading edge of invasive bladder cancer. (B) ACTN4 expression in scattered invading bladder cancer cells (level 4 expression). (C) ACTN4 expression in invading bladder cancer cells (level 3 and 4 expression). (D) Primary tumor with increased ACTN4 expression (white arrows) (level 4). Invading bladder cancer cells with increased ACTN4 expression (black arrows).

assay, and cell counts showed that ACTN4 suppression decreased the invasive ability of bladder cancer cells but increased their proliferation. Although ACTN4 is reportedly associated with cancer aggressiveness, the results of the MTS assay and cell counts were unexpected. We, therefore, evaluated changes in the phosphorylation of Akt, ERKs and STAT3, which are possibly associated with cell proliferation. The phosphorylation of ERK1/2 in T24 and KU19-19 cells was increased by ACTN4 suppression, but that of Akt and STAT3 did not appear to be affected (Fig. 6). These results suggest that ACTN4 suppression increases bladder cancer proliferation in part through ERK1/2 phosphorylation.

\section{Discussion}

Decreased cell adhesion and increased cell motility are necessary steps for the initiation of cancer cell metastasis, and actin stress fiber distribution in cancer cells changes when their 
A
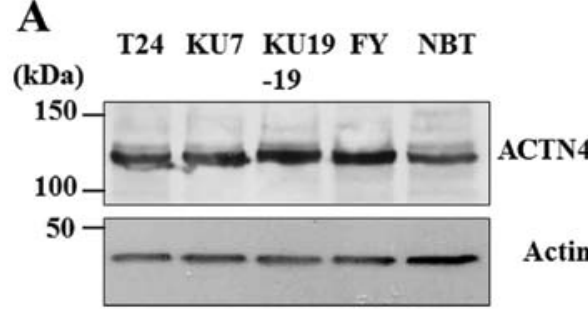

B

C

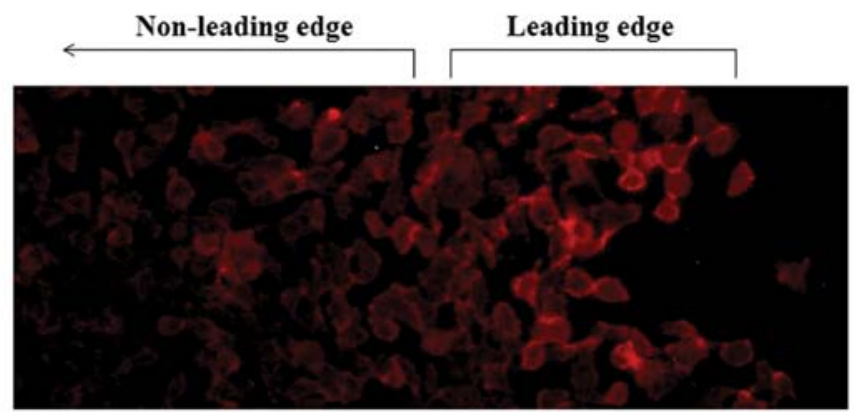

Figure 3. (A) Western blot analysis of ACTN4 expression in 5 human bladder cancer cell lines. All cell lines expressed ACTN4. (B) Western blot analysis revealed that ACTN4 expression was higher in the leading edge of cultured bladder cancer cells than in the non-leading edge of cultured bladder cancer cells (C) Immunocytochemistry indicated that ACTN4 expression was higher in the leading edge of cultured KU19-19 cells compared to the non-leading edge of cultured KU19-19 cells.

A

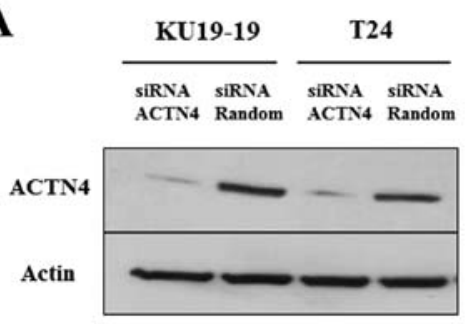

B

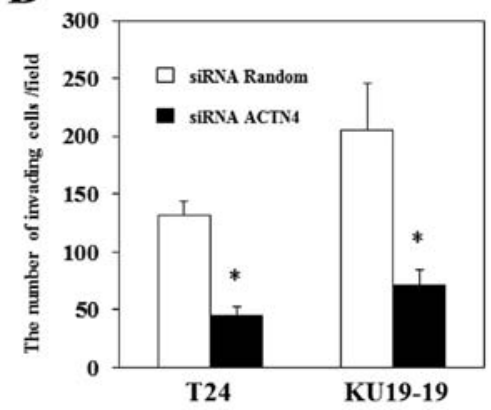

C T24

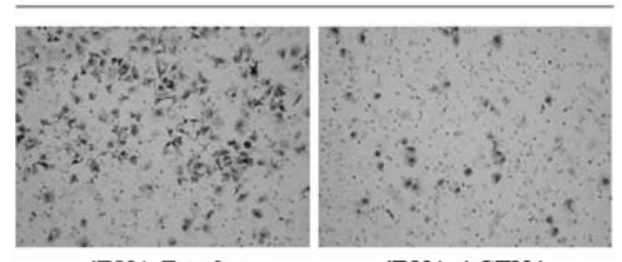

siRNA Random

siRNA ACTN4

KU19-19

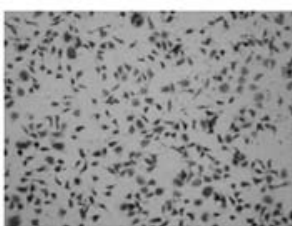

siRNA Random

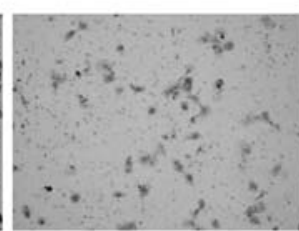

SiRNA ACTN4

Figure 4. (A) Western blot analysis showing that siRNA interference suppresses ACTN4 expression in T24 and KU19-19 cells. (B) Matrigel invasion assay results. The number of T24 cells invading through the chamber was significantly decreased by ACTN4 suppression (control siRNA, $131.6 \pm 11.9$ cells; ACTN4 siRNA, 45.6 \pm .5 cells) ( $\mathrm{p}=0.0495$ ). The number of KU19-19 cells invading through the chamber was significantly decreased by ACTN4 suppression (control siRNA, 205.2 \pm 40.4 cells; ACTN4 siRNA, 71.5 \pm 12.4 cells) $(\mathrm{p}=0.0209)$. (C) Representative images of the Matrigel invasion assay plates $(\mathrm{x} 100)$.

motility increases. ACTN4 was identified as a protein that was concentrated in the cytoplasm where colon cancer cells were sharply extended and thus was suggested to be associated with cancer cell invasion (10). In that report the increased cytoplasmic expression of ACTN4 was associated with poor prognosis of patients with breast cancer. Increased expression of ACTN4 in cancer tissues has been associated with poor prognosis in breast (10), ovarian (20), non-small cell lung (13) and pancreatic cancer (21). Increased ACTN4 expression in cancer tissues has been associated with higher percentages of lymph node metastasis in colon (14) and esophageal cancer (22). Although the above-mentioned studies indicate that ACTN4 promotes carcinogenesis, inverse findings were also reported for neuroblastoma (23) and prostate cancer $(24,25)$. Highly malignant neuroblastoma stem cells showed decreased growth ability and loss of tumorigenicity after transfection with ACTN4 cDNA (23). ACTN4 expression was downregulated in prostate cancer cells when compared with normal prostate 

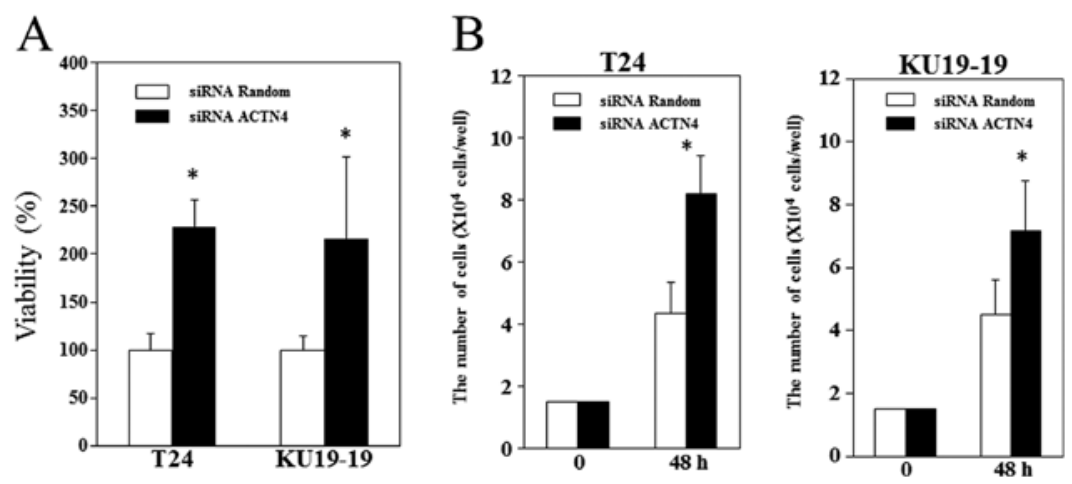

Figure 5. (A) MTS assay. Cell viability was significantly increased by ACTN4 suppression in T24 (229\% increase, p=0.0039) and KU19-19 cells (216\% increase, $\mathrm{p}=0.0163$ ). ${ }^{\mathrm{p}}<0.05$, compared with the control siRNA-treated cells. (B) Cell counts $48 \mathrm{~h}$ after siRNA treatment. The number of ACTN4-siRNA-treated T24 cells $\left(8.2 \pm 1.2 \times 10^{4}\right.$ cells/well) was significantly higher than the number of control-siRNA-treated T24 cells $\left(4.3 \pm 1.0 \times 10^{4}\right.$ cells/well), and the number of ACTN4 siRNA-treated KU19-19 cells $\left(7.2 \pm 1.6 \times 10^{4}\right.$ cells/well) was significantly higher than the number of control-siRNA-treated KU19-19 cells $\left(4.5 \pm 1.1 \times 10^{4}\right.$ cells/ well). ${ }^{*} \mathrm{p}<0.05$, compared with the control-siRNA-treated cells.

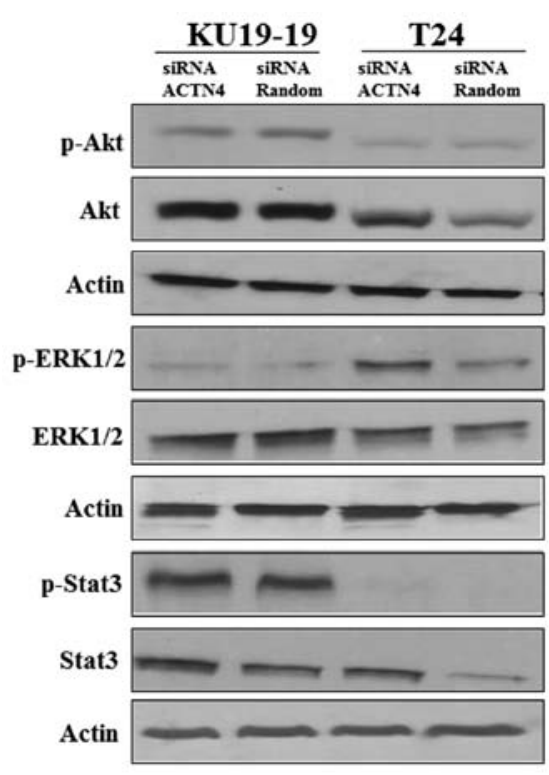

Figure 6. Western blotting of Akt, ERKs and STAT3 in T24 and KU19-19 cells after 48-h siRNA treatment. ACTN4 suppression increased the phosphorylation of ERK1/2 but did not appear to affect phospho-Akt and phospho-STAT3, suggesting that ACTN4 suppression increased bladder cancer proliferation in part through the ERK1/2 signaling pathways.

epithelial cells, and restoration of ACTN4 expression had a suppressive effect on the proliferation of prostate cancer cells (24). The association of ACTN4 expression with cancer aggressiveness thus differs between cancer types.

In our immunohistochemical analysis results, increased ACTN4 expression was associated with higher pT stage and higher histological grade. Although 3 of the 4 patients with pT4 disease had tumors predominantly showing low ACTN4 expression in the primary lesion, 2 of the 3 cases had cancer cells invading only into prostatic ducts and not prostatic stroma. Bladder cancer with only prostatic ductal invasion is considered to be less aggressive than that with prostatic stromal invasion. The 2 patients with pT4 disease who had only ductal invasion survived more than 5 years without metastasis. Another patient with pT4 disease who had a tumor predominantly showing low ACTN4 expression had a small region that showed high ACTN4 expression. This patient succumbed to disease within 1 year after radical cystectomy. Although the tumors in 34 of the 42 patients with invasive bladder cancer (81\%) showed high ACTN4 expression, the tumors in the other 8 cases showed low ACTN4 expression. Most of these 8 tumors, however, had small regions showing high ACTN4 expression. In these tumors, ACTN4 expression was particularly increased in tumor budding. Similar immunohistochemical results have been reported in colon cancer (14). The increased expression of ACTN4 was most significant in dedifferentiated cancer cells at the invasive front.

All bladder cancer cell lines expressed ACTN4 protein (Fig. 3A). Koizumi et al (15) reported that ACTN4 mRNA and protein expression levels were higher in bladder cancer cells than in cultured urothelial cells. In the present study the leading edge of the cultured bladder cancer cells (T24 and KU19-19) appeared to be stained more strongly for ACTN4 than the non-leading edge region, and western blot analysis supported these immunohistochemical results (Fig. 3B). In the immunohistochemical analysis for human bladder cancer tissues, high expression of ACTN4 was shown in the leading edges of bladder cancer (Fig. 2A). Furthermore, ACTN4 knockdown suppressed the invasive ability of both T24 and KU19-19 cells. The suppression of invasive ability by ACTN4 knockdown was also noted in J82 invasive bladder cancer cells (15) and BxPC3 pancreatic cancer cells (18). These results suggest that ACTN4 is involved in bladder cancer invasion. Activated forms of Rac1 and $\mathrm{Cdc} 42$ were found to upregulate ACTN4 in NIH3T3 cells, and Rac1 and Cdc42 may be upstream molecules of ACTN4 in cancer cell invasion (26). The upstream molecules that regulate ACTN4 in bladder cancer invasion need to be determined.

Notably, the proliferation of bladder cancer cells was increased by ACTN4 knockdown. Koizumi et al (15) reported that ACTN4 suppression increased the proliferation of J82 bladder cancer cells, and colony formation was inversely correlated with the amount of ACTN4 protein in A172 neuroblastoma cells that were ACTN4 stable transfectants (24). We, therefore, examined changes in the phosphorylation of major signaling molecules that can influence cell proliferation and 
found that ACTN4 suppression increased the phosphorylation of ERKs but not that of AKT or STAT3, suggesting that the increased proliferation of bladder cancer cells that was caused by ACTN4 suppression was at least partly mediated by the ERK pathway. Koizumi et al (15) suggested that Wnt signaling may also be associated with bladder cancer cell proliferation elicited by ACTN4 suppression. The signaling pathways by which ACTN4 suppression increases proliferation warrant further investigation.

In the present study, we examined whether high ACTN4 expression in resected tumors predicted poor prognosis in patients who underwent radical cystectomy and found that it was not an independent predictor of prognosis. A high percentage $(81 \%)$ of the patients with muscle-invasive bladder cancer showed high ACTN4 expression, and most of the patients ( 7 of 8 ) who had invasive bladder cancer but with low ACTN4 expression had minor components with high ACTN4 expression. In the present study we used the predominant ACTN4 expression level in each patient as that patient's ACTN4 expression level, and some patients whose bladder cancer showed predominantly low ACTN4 expression had components with high ACTN4 expression. The predominant ACTN4 level therefore did not always reflect the aggressiveness of each tumor. Because almost all patients with muscle-invasive bladder cancer $(97.6 \%)$ had variable amounts of bladder cancer tissues with increased ACTN4 expression, increased ACTN4 expression appeared to be an essential change in bladder cancer invasion. If increased ACTN4 expression is a basic change necessary for invasion, it is reasonable that high ACTN4 expression was not an independent predictor of prognosis in patients with invasive bladder cancer who underwent radical cystectomy. In addition, the results of our cell viability assay showed the possibility that ACTN4 expression suppresses cell proliferation in bladder cancer. In the 22RV1 prostate cancer cell line cell proliferation was inhibited by ACTN4 overexpression (24). If ACTN4 expression suppresses cell proliferation in bladder cancer, it may have a positive impact on patient prognosis even though it stimulates invasion.

In conclusion, in the present study, high ACTN4 expression was associated with a higher pathological stage and a higher histological grade but was not an independent predictor of prognosis in patients with invasive bladder cancer. Our immunohistochemical and in vitro results indicate that ACTN4 may play a fundamental role in bladder cancer invasion. ACTN4 expression stimulates the invasion of bladder cancer cells but may suppress their proliferation and produce conditions in which they invade easily. Further study will be needed to clarify the role of ACTN4 in bladder cancer invasion.

\section{References}

1. Ghoneim MA, el-Mekresh MM, el-Baz MA, el-Attar IA and Ashamallah A: Radical cystectomy for carcinoma of the bladder: critical evaluation of the results in 1,026 cases. J Urol 158: 393-399, 1997.

2. Stein JP, Lieskovsky G, Cote R, et al: Radical cystectomy in the treatment of invasive bladder cancer: long-term results in 1,054 patients. J Clin Oncol 19: 666-675, 2001.

3. Takahashi A, Tsukamoto T, Tobisu K, et al: Radical cystectomy for invasive bladder cancer: results of multi-institutional pooled analysis. Jpn J Clin Oncol 34: 14-19, 2004.
4. Rosenberg JE, Carroll PR and Small EJ: Update on chemotherapy for advanced bladder cancer. J Urol 174: 14-20, 2005.

5. von der Maase H, Hansen SW, Roberts JT, et al: Gemcitabine and cisplatin versus methotrexate, vinblastine, doxorubicin, and cisplatin in advanced or metastatic bladder cancer: results of a large, randomized, multinational, multicenter, phase III study. J Clin Oncol 18: 3068-3077, 2000.

6. von der Maase H, Sengelov L, Roberts JT, et al: Long-term survival results of a randomized trial comparing gemcitabine plus cisplatin, with methotrexate, vinblastine, doxorubicin, plus cisplatin in patients with bladder cancer. J Clin Oncol 23: 4602-4608, 2005.

7. Ito K, Fujita T, Akada M, et al: Identification of bladder cancer antigens recognized by IgG antibodies of a patient with metastatic bladder cancer. Int J Cancer 108: 712-724, 2004.

8. Chen YT, Scanlan MJ, Sahin U, et al: A testicular antigen aberrantly expressed in human cancers detected by autologous antibody screening. Proc Natl Acad Sci USA 94: 1914-1918, 1997.

9. Scanlan MJ, Chen YT, Williamson B, et al: Characterization of human colon cancer antigens recognized by autologous antibodies. Int J Cancer 76: 652-658, 1998.

10. Honda K, Yamada T, Endo R, et al: Actinin-4, a novel actinbundling protein associated with cell motility and cancer invasion. J Cell Biol 140: 1383-1393, 1998.

11. Araki N, Hatae T, Yamada T and Hirohashi S: Actinin-4 is preferentially involved in circular ruffling and macropinocytosis in mouse macrophages: analysis by fluorescence ratio imaging. J Cell Sci 113: 3329-3340, 2000.

12. Wang SE, Shin I, Wu FY, Friedman DB and Arteaga CL: HER2/Neu (ErbB2) signaling to Rac1-Pak1 is temporally and spatially modulated by transforming growth factor $\beta$. Cancer Res 66: 9591-9600, 2006.

13. Yamagata N, Shyr Y, Yanagisawa $\mathrm{K}$, et al: A training-testing approach to the molecular classification of resected non-small cell lung cancer. Clin Cancer Res 9: 4695-4704, 2003.

14. Honda K, Yamada T, Hayashida Y, et al: Actinin-4 increases cell motility and promotes lymph node metastasis of colorectal cancer. Gastroenterology 128: 51-62, 2005.

15. Koizumi T, Nakatsuji H, Fukawa T, et al: The role of actinin-4 in bladder cancer invasion. Urology 75: 357-364, 2010.

16. Bubeník J, Baresová M, Viklický V, et al: Established cell line of urinary bladder carcinoma (T24) containing tumour-specific antigen. Int J Cancer 11: 765-773, 1973.

17. Tachibana M, Miyakawa A, Nakashima J, et al: Constitutive production of multiple cytokines and a human chorionic gonadotrophin beta-subunit by a human bladder cancer cell line (KU-19-19): possible demonstration of totipotential differentiation. Br J Cancer 76: 163-174, 1997.

18. Kikuchi S, Honda K, Tsuda H, et al: Expression and gene amplification of actinin-4 in invasive ductal carcinoma of the pancreas. Clin Cancer Res 14: 5348-5356, 2008.

19. Horiguchi A, Sumitomo M, Asakuma J, et al: Leptin promotes invasiveness of murine renal cancer cells via extracellular signal-regulated kinases and rho dependent pathway. J Urol 176: 1636-1641, 2006.

20. Yamamoto S, Tsuda H, Honda K, et al: Actinin-4 gene amplification in ovarian cancer: a candidate oncogene associated with poor patient prognosis and tumor chemoresistance. Mod Pathol 22: 499-507, 2009.

21. Hayashida $\mathrm{Y}$, Honda $\mathrm{K}$, Idogawa $\mathrm{M}$, et al: E-cadherin regulates the association between $\beta$-catenin and actinin-4. Cancer Res 65: 8836-8845, 2005.

22. Hatakeyama H, Kondo T, Fujii K, et al: Protein clusters associated with carcinogenesis, histological differentiation and nodal metastasis in esophageal cancer. Proteomics 6: 6300-6316, 2006.

23. Nikolopoulos SN, Spengler BA, Kisselbach K, Evans AE, Biedler JL and Ross RA: The human non-muscle $\alpha$-actinin protein encoded by the ACTN4 gene suppresses tumorigenicity of human neuroblastoma cells. Oncogene 19: 380-386, 2000.

24. Hara T, Honda K, Shitashige M, et al: Mass spectrometry analysis of the native protein complex containing actinin- 4 in prostate cancer cells. Mol Cell Proteomics 6: 479-491, 2007.

25. Jasavala R, Martinez H, Thumar J, et al: Identification of putative androgen receptor interaction protein modules: cytoskeleton and endosomes modulate androgen receptor signaling in prostate cancer cells. Mol Cell Proteomics 6: 252-271, 2007.

26. Teramoto H, Malek RL, Behbahani B, Castellone MD, Lee NH and Gutkind JS: Identification of H-Ras, RhoA, Rac1 and Cdc42 responsive genes. Oncogene 22: 2689-2697, 2003. 